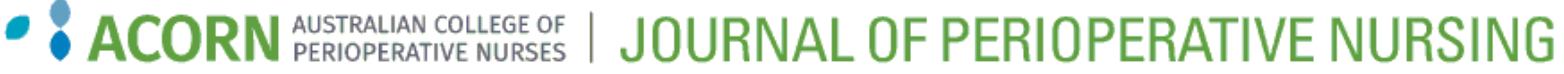

\title{
The value of guided operating theatre experience for undergraduate nurses
}

Follow this and additional works at: https://www.journal.acorn.org.au/jpn

Part of the Education Commons, Health Services Administration Commons, Health Services Research Commons, Perioperative, Operating Room and Surgical Nursing Commons, and the Surgery Commons (c) (i)

This work is licensed under a Creative Commons Attribution 4.0 License.

\section{Recommended Citation}

Foran, Paula (2016) "The value of guided operating theatre experience for undergraduate nurses," Journal of Perioperative Nursing: Vol. 29 : Iss. 1 , Article 1.

Available at: https://doi.org/10.26550/2209-1092.1000

https://www.journal.acorn.org.au/jpn/vol29/iss1/1

This Article is brought to you for free and open access by Journal of Perioperative Nursing. It has been accepted for inclusion in Journal of Perioperative Nursing by an authorized editor of Journal of Perioperative Nursing. 


\section{The value of guided operating theatre experience for undergraduate nurses}

\author{
The Australian population is ageing ${ }^{1}$ which, in turn, will lead to \\ an increased demand for surgical health services ${ }^{2}$. So too the \\ perioperative nurse matures, with over 50 per cent of the current \\ Australian perioperative nursing workforce being older than 51 \\ years; 41 per cent are between 51 and 60 years, and 15 per cent are \\ older than 60 years of age . $^{3}$
}

\section{Introduction}

As this workforce gets closer to retirement, and the profession looks ahead to cultivate replacements for the retiring staff, much research and discussion has focused on the relationship between lack of exposure by undergraduate nurses to the operating suite and a dwindling number of nurses entering this specialty field ${ }^{4-6}$. The difficulty lies in the ability to recruit new nurses into a specialty area that has been limited or completely excluded from the undergraduate nursing curricula?.

Another concern that has been voiced since the removal of perioperative nursing from most undergraduate nursing core curriculum worldwide is about the possible loss of surgical knowledge for student nurses. Questions have been raised regarding the ability of nurses to care for pre- and postoperative patients on the acute surgical wards ${ }^{8-10}$ A surgical patient's journey involves a three-part process, with pre-operative, operative and post-operative phases ${ }^{11}$. This research considered the potential educational impact on undergraduate and graduate nurses when the middle piece of the three-part surgical patient journey was omitted.

Can nurses who have not been involved in guided operating theatre experience care for patients before and after surgery with the same level of knowledge and expertise as those who have?

This paper will present an overview of a national mixed methods research project that investigated these of operating theatre education offered in Australia. Transferable skills learned via operating theatre experience that may be useful in the surgical wards will be explored and also possible correlations between students' experiences in this specialty area and recruitment and retention of staff to this specialist area.

\section{Literature review on surgical adverse events}

Timely, appropriate surgery and highquality pre- and post-operative care may be the key to preventing deaths in the first 48 hours after surgical procedures $^{12}$. There is consensus that adverse surgical events constitute a serious problem, killing more people annually than breast cancer or AIDS ${ }^{13}$. Surgical complications are estimated at between $50 \%$ and $75 \%$ of all adverse medical events ${ }^{14}$. In Australian literature an adverse event may be defined as 'an unintended injury or complication which resulted in disability, death or prolongation of hospital stay, and is caused by issues, comparing different models health care management rather than the patient's disease'15. An Australian study reported that in a post-surgical control group 390 adverse events were detected in 190 patients ${ }^{16}$. More recent research has reported that surgical adverse events occur in $3.6 \%$ of all hospital admissions, representing $65 \%$ of all reported adverse events ${ }^{17}$. These surgical adverse events were severe in nature and $41 \%$ were considered to have been preventable ${ }^{17}$.

Whilst the mortality in surgical patients has fallen in the last decade, mainly due to effective recognition and treatment of patient deterioration, failures in this process are still occurring ${ }^{18}$. A recent American study looked at unexpected deterioration of surgical patients on post-operative surgical wards over a 12-month period ${ }^{18}$. Findings revealed only $54 \%$ of patient deterioration was detected and reported by nursing staff during general patient care, $34 \%$ were detected by medical staff during routine rounds and the final $12 \%$ were detected from either rapid response or code blue calls ${ }^{18}$. The Australian National Consensus Statement on Essential Elements for Recognising and Responding to Clinical Deterioration reports that measurable physiological abnormalities occur prior to adverse events such as cardiac arrest and 
death and suggests that early recognition of changes in a patient's condition followed by prompt and effective treatment can minimise these adverse events ${ }^{19}$. The statement also suggests that education must be provided to guarantee that health care workers are suitably skilled in knowledge of appropriate patient observations, identification of clinical deterioration and have the appropriate emergency management skills to treat patients who are deteriorating ${ }^{19}$.

When perioperative nursing was removed from the nursing core curricula of many universities it was replaced by a 'follow-through' style of learning ${ }^{7,11}$. This allows students to care for patients pre-operatively in the surgical ward, observe the patient's surgery and subsequent recovery in the post-anaesthesia recovery unit and travel back to the surgical ward to care for the patient during the post-operative phase $e^{7,11}$. However, this is on an ad hoc basis (as it is dependent on the ability of the ward to release the students and the desire of the students to observe surgical procedures). Perioperative managers cannot foresee attendance by the students so the educational infrastructure afforded to the students who have formal university placements, which provides guided theatre experience, is not provided. In this scenario, staffing constraints and busyness of the operating theatre play a major role in the ability of the students to access quality supervision and education $n^{7,11}$.
Guided education is where students have been given explicit instructional guidance and where concepts and skills are explained ${ }^{20}$ by an experienced perioperative nurse. Non-guided learning, in the context of this research, is where an experienced perioperative nurse has not been allocated to assist the student" ${ }^{11}$. The most significant basis for workplace learning is the provision of access to guidance from more experienced others, and the lack thereof may well inhibit the ability to learn²1.

Education debate has been raging for over a century regarding the amount of guidance students require to learn ${ }^{20,22}$. On one side of this debate there are those who believe that all learners, novice and expert, 
learn more effectively when asked to interpret information for themselves by providing unguided or partially guided learning methods ${ }^{20}$. On the other side, some believe that experts and novices differ, whilst experts are able to learn effectively with minimal guidance, novice learners require more explicit instructional guidance to thrive $e^{22-25}$.

The cognitive load theory, as described by Sweller ${ }^{24}$ is based on the hypothesis that the human brain has two types of memory: shortterm, which has limited storage capacity, and long-term, which has almost unlimited capacity ${ }^{11}$. Whilst the working memory processes and stores information for a short time, the main aim of learning is to provide knowledge which will eventually be stored in the long-term memory for later use ${ }^{22}$. In nonguided or minimally guided learning great demands are placed on the working memory as it searches to find problem solutions; this work does not leave the working memory free to be available to contribute to the accumulation of knowledge in the long-term memory, thus limiting learning ${ }^{22}$. Novice learners, like undergraduate nurses in a foreign environment such as the operating theatre, are of great concern as the heavy demands that free exploration of complex environments place on the working memory may in fact be detrimental to learning ${ }^{20,22}$.

A gap in the research literature was noted in the exploration of the operating suite as an area of rich learning, and in the educational effects of limited or no operating theatre experience on undergraduate nurses' ability to care for pre- and post-operative patients in the surgical ward area. A second gap is noted in the comparison of students' experience in the operating suite and the impact of this on both recruitment of new graduates and retention of existing staff.

\section{Method}

Considering the changes to nursing curricula, concerns raised in the literature and evidence of adverse surgical events, there was value in investigating the following research questions. The three research questions that were addressed in this doctoral research were:

1. Do undergraduate nurses need to be involved in guided operating suite practical experience in order to achieve skills and knowledge that will support a high standard of nursing care in the pre- and post-operative surgical wards?

2. What are the different models of operating suite education offered to Australian undergraduate nursing students? Which of these models yields the best educational outcomes and what are the transferable skills acquired from operating suite experience that assist in pre- and post-operative surgical nursing care?

3. How might these differing models of operating suite education impact on recruitment and retention of nurses to this specialist area?

To facilitate the answer to Research Question 1, there needed to be a measure of surgical nursing knowledge designed to compare undergraduate nurses who had been involved in guided operating theatre practice experience and participants who had been involved in non-guided operating theatre practice experience. To facilitate this information on final semester student nurses' knowledge of pre- and post-operative care, a quantitative assessment tool was developed to provide data on the participants' knowledge level in these areas. Validation of this tool was undertaken by surgical ward nurses and expert peers. Data were also collected on the models of operating theatre education the students had been involved in (which included either guided or non-guided practice), allowing comparison between students' test scores and guided and non-guided practice.

Research Question 2 was addressed in three parts. The first part of the question asked about the models of operating suite education that were available in Australia for undergraduate nurses at the time of investigation. To elicit this information, a phone survey was conducted. All Australian universities that offered undergraduate nursing were contacted and asked about their undergraduate operating theatre education. From this information a taxonomy and selection criteria for invitation into the research were developed.

The second part of Research Question 2 asked if any of the operating theatre models were able to yield a better educational outcome than others. To facilitate this answer, statistical comparisons were made between participants' test scores and:

- the models of operating theatre education that the participants were involved in

- the education pattern mix students participated in

- the time spent in the operating suite.

The third part of Research Question 2 inquired about transferable skills that may have been learned during operating theatre education. To facilitate this investigation, qualitative data were collected in the form of short answer questions that asked participants about possible 
skills learned in the operating suite that would assist their nursing care outside the theatre environment.

In answering Research Question 3, which focused on the recruitment and retention of nurses to this specialist area, recruitment of new nurses to the operating suite was addressed by asking participants if they had enjoyed their time in the operating suite, and if they would consider working in this specialty area after graduation.

Retention of nurses within the operating suite involves the ability to retain staff already working in the field. The possible impact that the different models may have on staff supervising students in the operating suite was addressed via a focus group of experienced operating theatre nurses educators.

The model of education referred to the mode of delivery and curriculum placement of the subject (where the subject sat in the curriculum) and included core curriculum (where all students participated), elective, online and mixed (a core curricula subject often called 'high dependency' where students choose between clinical placements of which one was perioperative nursing) $)^{7,11}$. Students from universities who did not have a core curriculum subject and did not choose perioperative nursing made up the final model of 'no formal OR education'7,11. These students may have had the opportunity to have a 'follow through' visit to observe their patient's procedure and subsequent recovery before returning with the patient to the unit,11. Unfortunately, as these visits are on an ad hoc basis, perioperative managers have no ability to allocate staff to formally precept these students; thus the student learning is non-guided.

The different teaching and learning opportunities offered within the model of education were referred to as the pattern mix. Included in this were a combination of operating theatre nursing theory (theory); guided practical workplace experience (guided practice); non-guided practical workplace experience (non-guided practice), and extra practical experience (extra experience). Extra experience was recorded where students hours were more than had been arranged by their university as students had made personal arrangements with the operating theatre management to have extra time.

Ethics approval was sought and granted from the Deakin University Human Ethics Advisory Group.

Quantitative statistical analyses were conducted using both SPSS version 20.0 for Windows (SPSS Inc, Chicago) and XLStatistics 2008. When assessing differences between only two groups an independent t-test analysis was conducted ${ }^{11}$. For assessment of differences between more than two independent groups and where the assumptions associated with the application of parametric statistical methodologies were met, overall differences were identified using a one-way analysis of variance (ANOVA) ${ }^{11}$. As ANOVA is an omnibus test statistic and does not indicate which specific groups were significantly different from each other, subsequent post-hoc analysis was conducted using Tukey's Honestly Significant Difference (HSD) test $^{11}$. Where assumptions were not met, the nonparametric analogue to the ANOVA, the Kruskal-Wallis rank test was employed, with subsequent post-hoc analysis conducted using the Mann-Whitney test ${ }^{11}$. All posthoc analysis probabilities were adjusted for with the Bonferroni test $^{11}$. In addition to ANOVA when making comparison between time spent in theatre and participant score, a Pearson product-moment correlation coefficient was computed and a linear regression model was constructed $^{11}$. Qualitative data were manually coded and themed and validation of emergent themes was undertaken by a group of expert peers $^{11}$.

The research data were collected in four phases. These were:

Phase 1: Information was sought from all Australian universities that offered undergraduate nursing inquiring about their learning opportunities in operating theatre nursing.

Phase 2: Final year undergraduate nursing students in their final semester of university were involved in both quantitative and qualitative data collection.

Phase 3: Curriculum coordinators of participants involved in Phase 2 were involved in qualitative data collection (this data has not been reported in this paper).

Phase 4: Follow-up study - Nurses following their first year of practice were tested to compare the knowledge from graduating student to graduate nurse.

\section{Findings}

\section{Answering Research Question 1}

Research Question 1 asked if undergraduate nurses needed to be involved in guided operating suite practical experience in order to achieve skills and knowledge that support a high standard of nursing care in the pre- and post-operative surgical wards.

The t-test analysis was used to compared students who had participated in guided practical $(n=161)$ experience with students who had non-guided practical experience $(n=172)$. Undergraduate nurses who were involved in guided practice 
participated in the following pattern mixes:

- theory, guided practice and extra experience

- theory and guided practice

- guided practice.

Undergraduate nursing students who were involved in non-guided practice participated in the pattern mixes of:

- theory and non-guided practice

- theory

- non-guided practice

and

- nothing (neither theory nor any form of practice).

An independent t-test was used to compare differences between scores of those who had been exposed to guided and non-guided practical experience ${ }^{11}$. The t-test analysis revealed a $p$ value of $<0.001^{11}$. From these findings, the conclusion can be made that there was a statistically significant difference in the knowledge levels between the two groups $(t=4.743 ; p<0.001)^{11}$. Students who have been exposed to guided operating theatre experience had achieved a higher score on the questionnaire (which tested knowledge regarding surgical ward nursing $)^{11}$.

The concept of pass/fail is important in nursing as it indicates the participants' ability to not only progress further academically, but also determines their ability to gain nursing registration ${ }^{11}$. Results revealed a statistical significance between pass/fail and guided and non-guided experience $(t=4.304$ $p<0.0001)^{11}$. The pass rate was set at 10 out of 20 .

Data revealed a pass rate of $76 \%$ for the guided practical experience group and $56 \%$ for the non-guided practical experience group (Figure 1).
As much learning is achieved during a graduate nurse program, nurses were retested following this extra educational year.

Results revealed nurses who received guided operating experience as either an undergraduate or graduate nurses had a $100 \%$ pass rate ${ }^{11}$ (Figure 2), revealing possible consolidation of their undergraduate learning. Those who had non-guided or no operating theatre experience had a 53\% pass rate $^{11}$. The statistical significance was $(t=6.461 p<0.0001)^{11}$.

\section{Answering Research Question 2}

The first part of Research Question 2 asked about the different models of education that were offered to Australian undergraduate nursing students.

At the time of data collection (2009), 31 Australian universities offered undergraduate nursing degrees and of these 13 offered specific operating theatre nursing education $n^{7,11}$. In choosing models of education that would be invited to participate, a set of selection criteria were developed. This criteria observed: innovation of model; geographical location of university (a national project required a spread across several states); differing models of perioperative education; and specific theatre education as opposed to surgical ward nursing education ${ }^{7,11}$. From the six participating universities, there were the following models: one
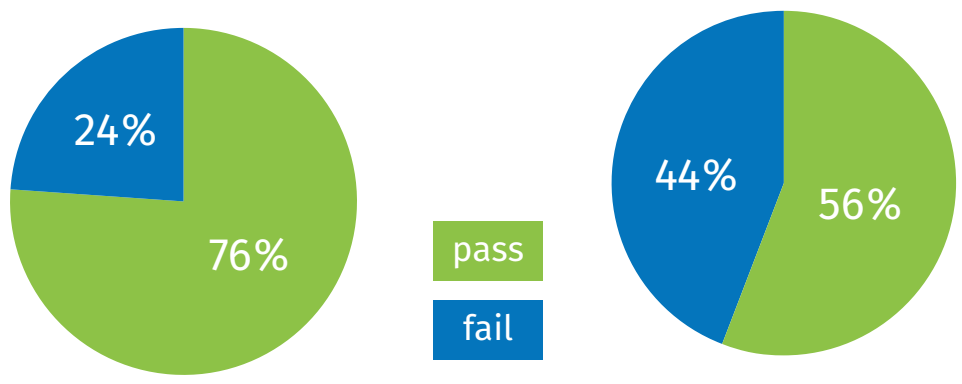

Figure 1: Pie chart depicting pass/fail between undergraduate nurses in guided groups (at left) and non-guided groups (at right) elective model; one online model; two core curriculum (all students participated) models; and two mixed (subject fell within the core curricula but not all students could participate $)^{7,11}$.

The second part of Research Question 2 asked which of these models yielded the best educational outcomes.

A one-way ANOVA revealed a statistical significance ( $F=4.836$, $p<0.01$, effect size 0.06$)$ in comparison of student score and education model ${ }^{11}$. Post-hoc testing using a Tukey (HSD) revealed that the statistical significance $(p<0.006)$ was between the two groups at the highest and the lowest points of score, namely the 'elective' model (which provided theory prior to 40 hours of guided experience) and 'no formal operating theatre education' (which provided non-guided or no practical experience) $)^{11}$.

A Kruskal-Wallis rank test was performed with post hoc testing using a Mann-Whitney Mu test to compare student score and pattern mix ${ }^{11}$. This revealed the only two groups that were statistically significantly different from each other $(p<0.001)$ were 'theory and guided practice' and 'nothing'. a positive gradient $(p<0.001)$, as a consequence of increased

\section{fail}

A regression analysis revealed indicating an increased test score 

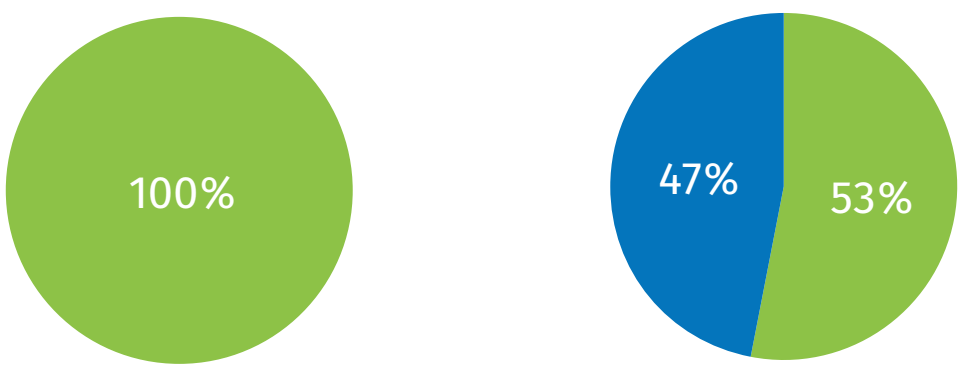

Figure 2: Pie charts depicting pass/fail between graduate nurses in guided groups (at left) and non-guided groups (at right)

time ${ }^{11}$. The overall findings from the correlation between time in the theatre and participant score showed a statistically significant relationship, revealing that time is advantageous to score ${ }^{11}$. Subsequent analysis indicated that time was advantageous up to about 40 to 60 hours and then seemed to plateau, further indicating that there may be diminishing return on effort after about 60 hours in the undergraduate nursing cohort"11.

The third part of Research Question 2 asked about the possible transferable skills students may have acquired from operating suite experience that assisted them in pre- and postoperative surgical nursing care.

Initially, participants were asked if they had learned any skills that they could use on the surgical wards.

It is worth noting that of the 68 participants who chose 'not stated', 61 did not have any operating theatre experience (Figure 3).

Secondly, participants were asked to state the skills that they learned. The transferable skills reported by the participants were grouped into nine themes, which included:

- pre- and post-operative care

- pain management

- anatomy and physiology

- what the patient goes through

- surgical procedures
- patient education

- asepsis

- technical skills and patient assessment.

Some qualitative comments on transferable skills included:

\section{Participant 15}

'It provided insight into what a patient goes through during a procedure and how it takes its toll on the body - this enhances my understanding and increased my knowledge in post-op care in the wards'

\section{Participant 315}

'Yes by watching the procedure it allowed me to understand why it is important to do observations and all post-op care'

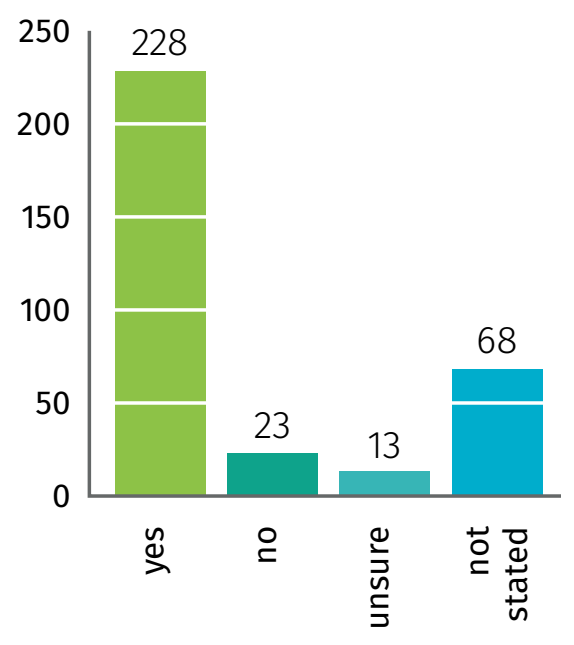

Figure 3: Bar graph showing participants' responses about transferable skills

\section{Participant $\mathbf{5 8}$}

'Yes. I learned why patients may be so sore because I watched their procedures so I have more of an understanding of what they were going through. It also gave me the skills to prepare them for theatre procedures'

\section{Participant 63}

'I felt more confident in explaining procedures to patients in the ward both pre-op and post-op. More aware of what they had been through'

\section{Participant 313}

'Yes, definitely in relation to asepsis/ infection control, plus it is great to know what particular procedures involve and wounds - made me mindful of infections'

\section{Participant 53}

'The skills learned in PACU, for example, patient assessment, I will definitely take with me'

\section{Answering Research Question 3}

The final research question asked about how the differing models of operating suite education impact on recruitment and retention of nurses to this specialist area.

To observe the impact on recruitment, participants were asked if they enjoyed their time in the operating theatre and if they would consider future employment in the operating theatre following graduation. Answers were compared to participants from guided and nonguided practical operating theatre experience.

Students who were provided with guided operating theatre practice were more likely to enjoy their experience (Figure 4).

Participants who were provided with guided practical experience in the operating theatre were more likely to consider perioperative nursing as a career, revealing that guided perioperative practice for 


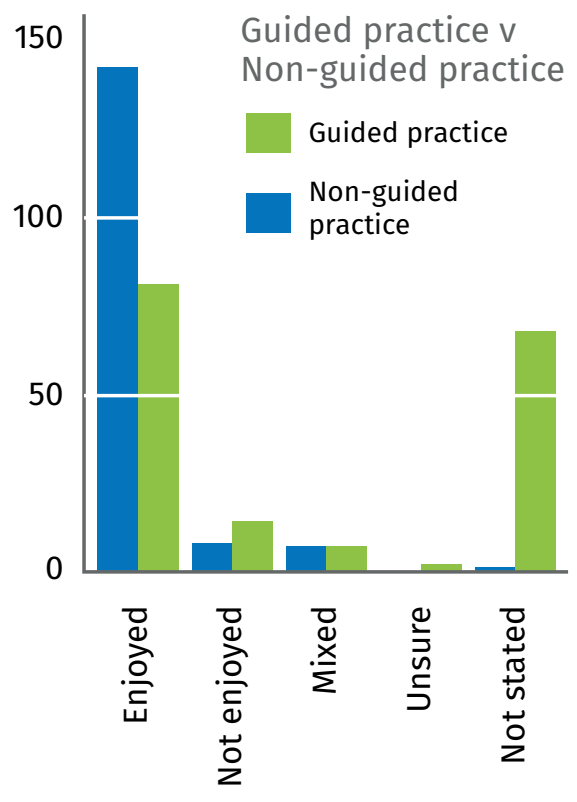

Figure 4: Enjoyment and guided versus non-guided practice

undergraduate nursing students' is a positive variable in enjoyment of the placement and does play a part in recruitment of nurses to this specialist area (Figure 5).

To investigate student nurses' impact on retention of the experienced staff supervising the students, a focus group of 22 experienced OR educators was convened and asked if they had noted connections between the different models of education that undergraduate nurses visiting their operating suites were exposed to, and any subsequent effects the different models played on the perioperative workforce that impacted on the retention of staff. Participants all stated a fear of unprepared visitors accidentally causing possible harm to patients by contaminating sterile instruments? Participants related stories of this occurrence?. One participant's comments resonated the sentiments of the group:

\section{Participant 3}

'Unprepared students are a huge stressor to our staff and a reason why we have lost staff. This is certainly the comments we hear from our undergraduate educators who have students from one university (unprepared with no unit offered), compared to another where students are offered a formal subject. All students are offered the same level of support when they arrive at our operating suite - but those from universities that offer a specialty unit need less and flourish quickly'7,p.259

\section{Discussion}

Findings from this research, conducted nationally in Australia, support previous research conducted in one Australian state ${ }^{8}$, and in one university in America ${ }^{26}$, suggesting that there is great value in operating theatre experience to enhance the educational preparation of surgical ward nursing skills. This research differed from its predecessors by its national status, its participants being from different universities, and the direct comparison of students who were exposed to guided practical operating theatre experience as opposed to those who were exposed to non-guided experience or no experience at all.

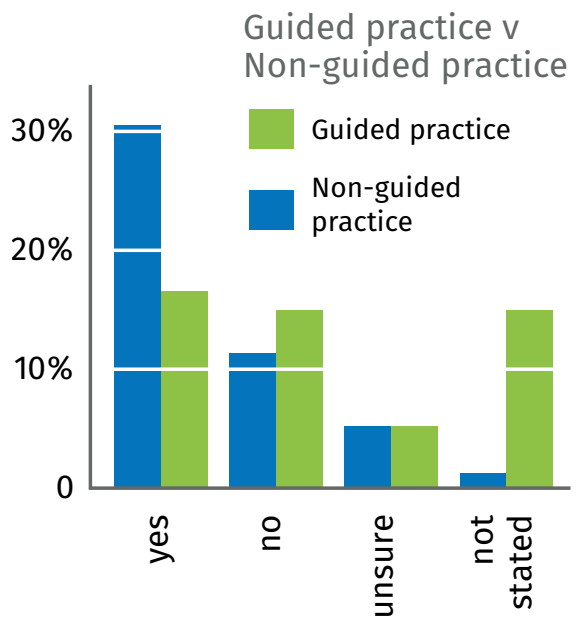

Figure 5: Future employment and guided versus non-guided practice
Surgical ward nursing knowledge was deficient in $44 \%$ of nurses at the time of their graduation and in $47 \%$ at completion of their Graduate Nurse Program year ${ }^{11}$. This reveals opposing principles when we note that operating theatre clinical experience has been gradually phased out of most nursing schools' curricula, even when this experience has been shown to provide greater surgical ward knowledge than what is gained from surgical ward nursing experience alone ${ }^{11}$.

These poor educational outcomes for non-guided learning may be explained by the cognitive load theory. In non-guided or minimally guided learning in complex environments like the operating theatre, great demands are placed on the working memory as it searches to find problem solutions; this work does not leave the working memory free to be available to contribute to the accumulation of knowledge in the long-term memory, thus limiting learning 22 .

Findings of this research have also shown that $18.3 \%$ of participants did not set foot in an operating suite in their entire undergraduate education and a further $9.7 \%$ had five hours or less ${ }^{11}$. This tells us that $28 \%$ of Australian undergraduate nurses who participated in this research spent less than half a day in the operating theatre during their undergraduate degree preparation ${ }^{11}$. Findings revealed in this research have shown that this is not sufficient to support safe knowledgeable pre- and postoperative surgical nursing care ${ }^{11}$.

Transferable skills that may be used in areas of nursing outside the operating theatre were learned whilst on a perioperative placement, which included knowledge on preand post-operative nursing care, the 
importance of taking observations and patient assessment skills, indicating that this area is one of rich learning for all undergraduate nurses.

Surgical adverse events are not uncommon ${ }^{14}$ and recent research informed us that when investigating patients who deteriorated on postoperative surgical wards, only 53\% on this deterioration was reported by the nurses caring for the patients ${ }^{18}$. It is not unusual for patients to deteriorate during the recovery phase in the PARU, and in fact, detection of deterioration and taking corrective measures (as often occurs in respirations and blood pressure) are the purpose of this specialised room ${ }^{27}$. The ability to observe and assist experienced nurses who are making patient assessments and dealing with deteriorating patients in a controlled environment and learning when to summon urgent assistance was stated as being learned via guided operating theatre experience. This educational experience will allow nurses to be better equipped to deal with possible patient deterioration in the surgical wards.

When caring for a patient postoperatively, the patient is recovering from both a surgical intervention and an anaesthetic intervention. With these interventions come a myriad of possible complications, which nurses need to understand. With understanding comes the ability for early recognition and treatment, which may prevent poor outcomes in patient care.

Ninety per cent of guided participants and only $47 \%$ on nonguided participants enjoyed their placement in theatre. The most significant individual variable contributing to undergraduate nurses enjoying their time in the operating suite as well as considering future employment was whether they had guided OR practical experience or not.

It was the perception of experienced perioperative educators who participated in the research that a major factor affecting the retention of experienced theatre nurses who supervised students was whether undergraduate nurses were adequately prepared prior to visiting the operating suite. This research has shown that unprepared students visiting the theatres are a possible risk to patient safety and a stressor to supervising staff. A limitation of this research was not conducting interviews with staff who had chosen to leave employment in this specialist area. A recommendation of this research is to build on these findings, specifically investigating the causative factors (including educational impacts) that result in perioperative nurses leaving the operating suite.

Findings of this research have also highlighted the importance of guided workplace learning and, in particular, the process of nurse preceptorship? This important area is worthy of further investigation?. Another limitation of this research was not investigating the specific role of perioperative preceptors. Therefore a second recommendation from this research would be that further research be undertaken investigating the skill set required for successful preceptors and the necessary qualifications to achieve this goal.

This research has shown that nonguided follow-through visits, in their current format, do not support best learning outcomes, do not support recruitment of new staff, and are a negative factor in the retention of experienced staff already working in this area?. To address these problems, findings of this research would recommend a reintroduction of perioperative nursing to the core undergraduate curricula?.

Rather than trying to find additional time from within already crowded curricula, a portion of time and resources already allocated to surgical unit nursing could be reallocated to encompass a structured, guided perioperative experience ${ }^{7,11}$. This is a reasonable approach as operating suite experience has been shown to provide enhance surgical unit nursing knowledge and skills suitable for preparation of generalist nurses ${ }^{28}$. This would, in turn, enhance recruitment of new nurses to this vital specialist area and assist in retention of the current workforce ${ }^{7,11}$.

As the world population ages, the need for surgical services and, in turn, the need for perioperative nurses will grow?. Recruitment of new nurses is essential to order to provide replacements for retiring staff and the retention of the current experienced workforce is vital, not just to maintain the staffing levels required, but also to providing their expert guidance in experiential learning for more junior staff before they retire and their knowledge and expertise is lost to the profession?

\section{Conclusion}

This research has successfully addressed these three questions and helped to close the gaps initially noted in Australian and relevant overseas literature. It has shown that all undergraduate nurses do need to be exposed to guided operating theatre experience in order to achieve a high standard of nursing care in the pre- and post-operative surgical wards. 
One of the most poignant remarks that has stayed with me throughout my whole research journey was born from a conversation I had whilst presenting at a surgical conference canvassing for volunteers for my Master of Professional Education and Training study. As a young nurse provided me with postal details, he stated:

\section{'After graduation I struggled in the} surgical wards, until I had a stint in the theatre. It was like the missing piece of the puzzle was added, and suddenly it all made sense' ${ }^{7}$.

Although it is sometimes a difficult task to constantly impart our knowledge to more junior nurses in the clinical area, we can now take solace that this understanding is being utilised not only in the operating theatre but in the surgical wards. As the elderly of tomorrow, we should all look at this as a personal investment into our own future care.

With the birth of modern surgery in the early eighteen hundreds came the term 'operating theatre'. It was so named as it was built resembling a twentieth century lecture theatre, allowing visitors to observe surgical procedures. It is ironic that in modern times the operating theatre has lost much of its undergraduate nursing audience.

\section{This paper presented an overview} of findings from a doctoral thesis and two previous published research papers. For further details on methodology related to this research, please refer to references 7 and 19.

\section{Acknowledgement}

I would like to thank all the nurses who took the time to be involved in this research. It was greatly appreciated.

\section{References}

1. Karmel R, Gibson D, Anderson P, Wells Y \& Duckett S (2012). Care trajectories through community and residential aged care services: Disease effects. Ageing and Society, Vol. 32, No. 8, pp. 1337-59.

2. The Australian Bureau of Statistics (2016). Aging Population. Available from: www. abs.gov.au/ausstats/abs@.nsf/mf/3101.0.

3. The Australian College of Operating Room Nurses (2015). ACORN members' survey. ACORN Journal, Vol. 28, No. 3, pp. 28-36.

4. Castelluccio D (2012). Education for the future. American Operating Room Nurses Journal, Vol. 95, No. 4, pp. 482-90.

5. Allanson AM \& Fulbrook P (2010). Preparation of nurses for novice entry to perioperative practice: evaluation of a short education program. Australian College of Operating Room Nurses Journal, Vol. 23, No. 2, pp. 14-27.

6. Bull R \& Fitzgerald M (2004). The invisible nurse - behind the scenes in an Australian OR. American Operating Room Nurses Journal, Vol. 79, No. 4, pp. 810-23.

7. Foran P (2015). Effects of guided undergraduate perioperative education on recruiting novice RNs and retaining experienced RNs. AORN Journal, Vol. 102, No. 3, pp. 254-61.

8. Touzeau P (2005). Undergraduate perioperative experience: Do structured placements in the operating suite make a difference to pre and postoperative surgical nursing care? Deakin University.

9. Walker L (1998). Why nursing students need an allocation to operating theatres. Nursing Standard, Vol. 13, No. 4, pp. 44-5.

10. Long C, George S \& Gulledge S (1995). Implementing a baccalaureate perioperative nursing elective. American Operating Room Nurses Journal, Vol. 61, No. 2, pp. 372-6.

11. Foran P (2015). Undergraduate surgical nursing preparation and guided operating room experience: A quantitative analysis. Nurse Education in Practice. In Press.

12. Mullen R, Scollay J, Hecht G, McPhillips G \& Thompson A (2012). Death within 48h - Adverse events after general surgical procedures. The Surgeon, Journal of the Royal College of Surgeons of Edinburgh and Ireland, Vol. 10, pp. 1-5.

13. de Vries E, Ramratten M, Smorenberg S, Gouma D \& Boermeester M (2008). The incidence and nature of in-hospital adverse events: a systematic review. Quality Safe Health Care, Vol. 17, pp. 216-23

14. Pinney D, Pearce M \& Feldman S (2010). Detecting adverse events in dermatologic surgery. Dermatological Surgery, Vol. 36, pp. 8-14.

15. Kable A, Gibberd R \& Spigelman A (2002). Adverse events in surgical patients in Australia. International Journal for Quality in Health Care, Vol. 14, No. 4, pp. 269-76.
16. Bellomo R, Goldsmith D, Uchino S et al. (2004). Prospective controlled trial of effects of medical emergency team on postoperative morbidity and mortality rates. Critical Care Medicine, Vol. 32, No. 4, pp. 916-20.

17. Zegers $M$, de Bruijne $M$, de Zeizer $B$ et al. (2011). The incidence, root-cause, and outcomes of adverse events in surgical units: implication for potential preventable strategies. Patient Safety in Surgery, Vol. 5, No. 13, pp. 3-11.

18. Helling $T$, Martin L, Martin M \& Mitchell M (2014). Failure events in transition of care for surgical patients. Journal of the American College of Surgeons, Vol. 218, No. 4, pp. 731-3.

19. Australian Commission for Safety and Quality in Healthcare (2010). National consensus statement: essential elements for recognising and responding to clinical deterioration, pp. 1-20.

20. Clark R, Kirschner P \& Sweller J (2012). Putting students on the path to learning. American Educator, Spring, pp .6-11.

21. Billett $S$ (2001). Learning in the Workplace - Strategies for Effective Practice. Allen \& Unwin, p. 222.

22. Kirschner P, Sweller J \& Clark R (2006). Why minimal guidance during instruction does not work: an analysis of the failure of constructivist, discovery, problem-based, experiential, and inquiry-based teaching. Educational Psychologist, Vol. 41, No. 2, pp. 75-86.

23. Mayer RE (2004). Should there be a three-strikes rule against pure discovery learning? American Psychologist, Vol. 59, No. 1, pp. 14-9.

24. Sweller J (1994). Cognitive load theory, learning difficulty, and instructional design. Learning and Instruction, Vol. 4, pp. 295-312.

25. Vygotsky L (1978). Mind in Society - the development of higher psychological processes: Cambridge, MA: Harvard University Press.

26. Sigsby L \& Yarandi H (2004). A knowledge comparison of nursing students in perioperative verses other rotations. American Operating Room Nurses Journal, Vol. 80, No. 4, pp. 699-707.

27. Drain C \& Odom-Forren J (2008). Perianesthesia nursing: a critical care approach $5^{\text {th }}$ ed. Philadelphia: Elsevier Saunders.

28. Gregory S, Bolling D, Langston N. Partnerships and New Learning Models to Create the Future Perioperative Nursing Workforce. (2014), AORN J. 99(1):96-105. 